\title{
Moving towards Personalized Geospatial Queries
}

\author{
Giorgos Mountrakis ${ }^{1}$, Anthony Stefanidis² \\ ${ }^{1}$ Department of Environmental Resources Engineering, State University of New York College of Environmental \\ Science and Forestry, Syracuse, USA \\ ${ }^{2}$ Center for Geospatial Intelligence, Department of Geography \& Geoinformation Science, George Mason University, \\ Fairfax, USA \\ E-mail: gm@esf.edu,astefani@gmu.edu
}

Received July 3, 2011; revised August 8, 2011; accepted August 10, 2011

\begin{abstract}
Geospatial datasets are typically available as distributed collections contributed by various government or commercial providers. Supporting the diverse needs of various users that may be accessing the same dataset for different applications remains a challenging issue. In order to overcome this challenge there is a clear need to develop the capabilities to take into account complicated patterns of preference describing user and/or application particularities, and use these patterns to rank query results in terms of suitability. This paper offers a demonstration on how intelligent systems can assist geospatial queries to improve retrieval accuracy by customizing results based on preference patterns. We outline the particularities of the geospatial domain and present our method and its application.
\end{abstract}

Keywords: Geospatial Databases, Geographic Information Systems, Geospatial Queries, Similarity Learning, Preference Modeling, Adaptive Systems, Digital Government.

\section{Introduction}

Geospatial information enjoys an increasingly important role in modern day societies, as it is used to support a variety of activities ranging from long-term planning and modeling, to emergency response and disaster management. Geospatial datasets may be diverse in nature, ranging from digital imagery and raster datasets to thematic layers of geographic information systems (GIS), vector data, and diverse sensor feeds. These datasets are collected, stored, and distributed by a variety of federal (e.g. the National Geospatial-Intelligence Agency-NGA), state (e.g. various state GIS offices), or local (e.g. town records) agencies. In addition to these authoritative datasets we are now witnessing the emergence of volunteered and participatory GIS [1], with datasets collected and contributed by non-profit organizations (e.g. Ushahidi) or even individuals. Through advancements in sensor technology, computer hardware, and software we have now reached the point where massive amounts of diverse types of geospatial datasets are integrated in distributed petabyte-size archives.

As the applications that use geospatial datasets are quite diverse, it is not rare to have the same dataset (e.g. a specific GIS layer) accessed by different users to sup- port diverse applications (e.g. location-based services through cell phone apps), or decision-making activities (e.g. land use modeling, crisis monitoring). In order to support query-based information retrieval (IR), geospatial datasets are indexed with metadata describing their essential properties (e.g. date, scale, accuracy, resolution, time, provider). Queries are typically performed by having a user stating his/her preference in terms of these metadata, e.g. "retrieve satellite images of Fukushima, Japan after March 11 2011, with pixel resolution equal to or better than 1 meter". The suitability of available datasets is then evaluated using standard distance metrics to compare them to the query request and rank them according to their similarity to the query parameters [2]. However, standard IR approaches fail to capture preference differences among diverse users. For example, a transportation expert and an emergency responder may have different preference patterns as they aim to retrieve satellite imagery depicting an area of interest at a specific instance. In the above-provided example relating to the Fukushima earthquake and subsequent tsunami, the transportation expert would prefer the most recent image available after the earthquake (e.g. from May, 2011), as her task may be to update the road network maps and capture the current state of transportation infrastructure 
in the area. On the other hand, the emergency responder may be interested in imagery showing the tsunami at its farthest location, before it started receding, to better assess the full extent of the impact area. For this responder the most recent imagery may therefore be of lesser value than imagery captured one to two hours after the earthquake.

The above is a simple example that shows potential variations in user-database interaction. Users that attempt to access collections of geospatial datasets have diversified information needs, reflecting differences in the experience and/or task at hand. As the user community of geospatial information is growing and becoming increasingly more diverse, such preference variations are becoming the norm rather than an exception. Standard IR techniques fail to take into account such complex preference patterns. In order to overcome this shortcoming we need to support the customization of user query execution by taking into account the particularities of a user's preferences. In this paper we present our approach to model user preferences in geospatial applications in order to improve the performance of geospatial queries. Before we proceed to the specifics of our method, we address trends in the generation, storage, and delivery of geospatial information.

\section{Geospatial Dataset Availability}

Authoritative geospatial datasets have been traditionally generated, used, and delivered by a variety of local, state, and federal government agencies. They are typically available as distributed collections through corresponding portals.

A representative example of a federal collection of geospatial datasets is the National Atlas (http://www. nationalatlas.gov/), offering map coverage across the US, with various themes (e.g. agricultural and transportation data overlaid on basic maps). The National Geospatial-Intelligence Agency (NGA, www.nga.mil) is offering access to charts and images. Users can access this information through its Raster Roam interface. Users can access a specific file either by selecting an area in a map display, or by using geographic names in a gazetteer-like approach. Furthermore, the Federal Geographic Data Committee (FGDC) (www.fgdc.gov) of the US Geological Survey (USGS) offers a distributed discovery mechanism comprising regional clearinghouses for digital geospatial dataset delivery. The Environmental Protection Agency (EPA) offers a wide variety of geospatially-referenced information (e.g. water quality and hazardous waste data), queried through a zip-code based system (http://www.epa.gov/enviro/html/qmr.html). In an effort to address the particular needs of disaster response, government agencies have set up dedicated portals that aggregate specific types of information. For example the Geospatial Multi-Agency Coordination Group effort (GeoMAC) (http://www.geomac.gov) aggregates firerelated information (incl. fire perimeter, terrain, and MODIS satellite datasets) across the continental United States. The Natural Hazards Support System (NHSS) ( http://nhss.cr.usgs.gov) is another example of an integrative portal, offering information on various natural hazards, e.g. volcanic, earthquake, and flooding information, together with satellite imagery. These federal-level datasets are complemented by countless regional collections of geospatial datasets collected and distributed through states, cities, and municipalities.

This early model of government-driven geospatial dataset collection and administration evolved through the proliferation of commercial remote sensing and geospatial analysis endeavours. For example, TerraServer (www.terraserver.com) is a collaboration of commercial (Microsoft, Compaq) and federal (USGS) partners, that offers a collection of digital imagery from numerous providers, arranged by location (e.g. coordinates, city name, street address, zip code), in various time instances. USGS photography comprises few terabytes of data, and is accessible through several host servers. Another notable commercialized collection of geospatial datasets is Mapquest (www.mapquest.com), using maps of the complete US in a variety of scales (e.g. 1:100,000, 1:25,000), 1-meter resolution aerial photography, and detailed street maps. Probably the most popular commercial implementation is the adaptation of Keyhole technology to build Google Earth (earth.google.com), aggregating a massive collection of satellite and field data.

The latest evolution of geospatial dataset availability is the on-going emergence of volunteered and participatory geographic information (VGI). Crisis mapping is a particularly relevant example, with the aggregation of authoritative datasets with contributed multimodal information to capture the consequences and evolution of a catastrophic situation [3,4]. Ushahidi (http://ushahidi. com) and its utilization during the Haiti 2010 earthquake disaster is by now a classic example of VGI at work. In addition to these examples, where the general public is contributing information, we also have services like Google MapMaker (http://www.google.com/mapmaker), where citizens are given the opportunity to perform information extraction tasks, like road centerline delineation, thus contributing directly geospatial information.

Thus we see that geospatial datasets are made available in numerous distributed collections of terabyte-sized archives of government, commercial, or non-profit agencies, each following established standards and specifica- 
tions in terms of a variety of parameters, including accuracy, format, metadata, scale, organization. Users access these datasets through the corresponding agency portals, either by browsing collections, or by forming metadata-based queries as mentioned in the previous section. The challenge faced by applications employing geospatial databases is to support the diverse needs of various users that may be accessing the same dataset for different applications. In order to do so we need to be able to take into account the complicated patterns of preference that correspond to a user and/or application, and use these patterns to rank existing datasets in terms of suitability.

To help our paper readability, here are the definitions of three important terms as presented within the context of this paper:

- Similarity refers to how appropriate is a given response to a geospatial information request.

- Preference relates to users expressing their individual suitability metrics for similarity.

- Similarity learning is the process of identifying and expressing in mathematical terms user preference on suitability.

\section{Similarity in Geospatial Information}

Before we get into the specifics of our similarity learning approach, let us first examine the information retrieval process and the corresponding steps involved. Every request for geospatial information involves a collection of methods, some of which have been addressed extensively in the literature and some others are newly investigated. In Figure 1, a schematic representation of the query process is shown. The following steps take place:

1) Users request an information object from the database (or more than one). For example a user may request all buildings within a given area that are larger than 2000 $\mathrm{m}^{2}$ and within $1 \mathrm{~km}$ from a highway exit.

2) Their request is translated into a structured query that the system understands and that is compatible with the database collection. In this step the user-provided information is matched to specific database fields and content. For example, ontology may be used to match the query for "buildings", a non-existent term in the database, to "single-family detached houses", an existent field in the database and therefore resolve ambiguity.

3) A query language is used as a mediator between user and database. This step is essential to convert user preferences into an automated executable code for information retrieval. One predominant example of such programming language is Structured Query Language (SQL).
4) Based on user request an indexing mechanism is used to return all potentially similar objects, in essence filtering dissimilar ones to accelerate the retrieval process. One filtering example is to temporary identifying all buildings larger than $2000 \mathrm{~m}^{2}$ and ignores all other buildings.

5) On this filtered object collection a similarity algorithm with properties extracted from a knowledge base is applied. The output is either a certain number of best answers (e.g. 10 best datasets) or answers within a specific similarity range (e.g. higher than $80 \%$ ). For further information see section 6 of this paper.

6) The results are presented to the users to assess their similarity accuracy.

In the above information flow there are several areas of interest that the database community is working on. Various disciplines are involved in the process and many different approaches have been proposed. Specifically, large distributed information source repositories are created and issues related to storing and accessing these databases are investigated. Ontologies are introduced to compensate for different field descriptions, as well as multi-node architectures and theoretical database models to support them. Query languages and indexing mechanisms for faster information retrieval are developed.

Our work concentrates on step five on the previous list. The goal is to develop a similarity algorithm that will rank the results in an accurate way. In order to do so, when a user is performing a geospatial information request, some identification information of user preferences is forwarded to a knowledge base (dotted arrow on the graph) and the appropriate similarity profile is extracted and incorporated in the query process.

The current methodologies used for similarity assessment of geospatial information have a common characteristic: they are non-adaptable to specific user preferences, instead they are expressed as pre-defined similarity measures and remain the same independently of task/user requirements. Similarity calculation is performed by storing geospatial information metadata as points in the feature space and using a distance metric to measure correlation to these points [5-7]. As mentioned above, commonly used metadata information includes expressions of resolution, accuracy, spatial extent, scale, date, and source. Usually a Minkowskian p-distance [8] is employed to define the similarity measure and is defined as:

$$
L_{p}(\bar{x}, \bar{y})=\left(\sum _ { i = 1 } ^ { n } \left(x_{i}-y_{i}\left({ }^{p}\right)^{1 / p}\right.\right.
$$

For $p=2$ we have the traditional Euclidean distance metric. If $p=1$ then the Minkowskian distance expresses the Manhattan distance function. Another function is the 


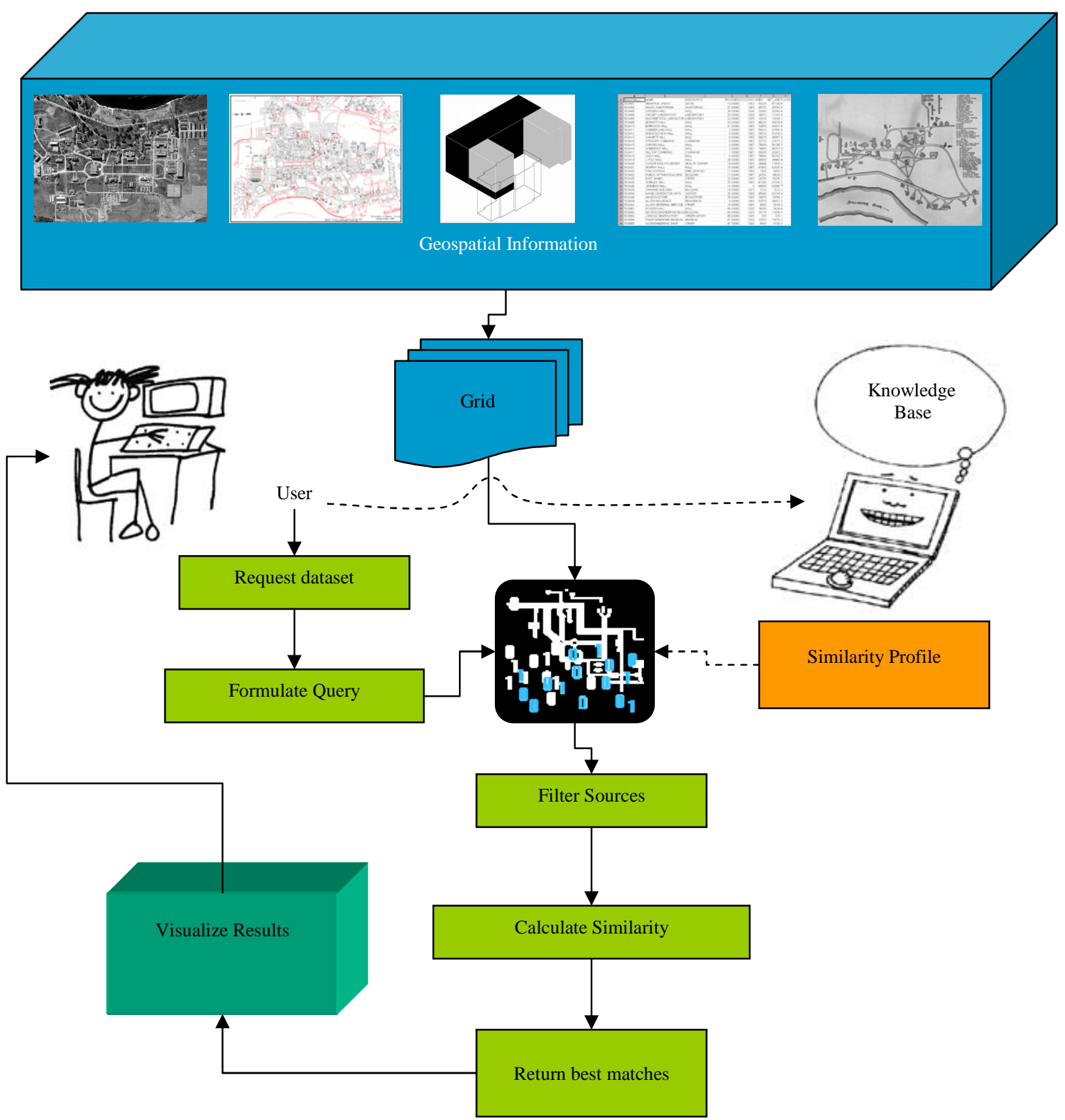

Figure 1. Query processing for geospatial information access.

Quadratic distance that is a weighted form of the multi-dimensional Euclidean. Other functions and corresponding mathematical expressions can be found in [9]. The above functions provide a simple model that allows efficient indexing by dimensionality reduction techniques. On the other hand though, this simplicity makes it impossible for these functions to take into account complex patterns of preference of diverse users, and use them to rank query responses accordingly.

So why not develop adaptable similarity methods specifically designed for geospatial databases? After all, this has been an active field of research for decades in other domains (e.g. text retrieval, web mining). The benefits of such work are obvious, but are the task simple enough to undertake? A major reason why adaptable similarity models for geospatial information have not yet progressed significantly comes from the considerable challenges imposed by the nature of the problem.

\section{Similarity Modeling for Geospatial Data: Not that Easy after All}

Adaptable similarity models for geospatial data impose a dual difficulty. The first comes from the multiple disci- 
plines involved in the similarity learning task and it is not unique to geospatial datasets. The second difficulty rises from the particularities of the geospatial domain and corresponding user needs. This uniqueness of the geospatial domain is the focus of our attention.

\subsection{The Interdisciplinary Nature of Similarity Learning}

Three general areas of research have been suggested in [10], namely psychology, data mining and machine learning. Briefly discussed here, the psychologists have concentrated on the human understanding and expression of similarity. Their research verifies that there is an objective parameter (i.e. user dependent), which has to be addressed for successful similarity modeling. Building on that, several tasks from the data mining field can be borrowed to accomplish our goal, tasks such as classification, regression, time series analysis and others. As expected, similarity learning naturally also falls under the general category of machine learning methods. The influence from multiple disciplines such as statistics, databases and artificial intelligence in machine learning is well-documented in the literature. From the similarity learning perspective an important distinction of machine learning algorithms is between supervised and unsupervised methods. Since most similarity learning algorithms learn from example, in other words they need a supervisor (teacher) to provide them some reference output values, they belong in the supervised category.

\subsection{Particularities of the Geospatial Domain}

Similarity learning in database queries is intrinsically connected with the data types stored. Geospatial data have important differences to online analytical processing data, general multi-dimensional data, traditional relational data or transactional data [11]. This uniqueness is partially attributed to the integrative nature of GIS. Many of the issues arise from the fact that geographic data span a wide range of perspectives and interests from the social to the physical aspects of the problem [12]. This mixture of perspectives coupled with the growing infrastructure for gathering information pose the following challenges:

1) Diverse data types. The wide variety of digital geographic data imposes a number of constraints/demands to similarity learning algorithms. Distributed datasets are becoming increasingly prevalent and important as a source of geographically referenced data [13] and thus tend to comprise a variety of geo-referenced multimedia data types, such as still and video imagery, text, graphics, and even audio and animations [14].

2) Dimensionality grouping and dependencies. Geo- spatial databases tend to be high-dimensional, as for example location information is accompanied by radiometric content, elevation data, ownership information, and temporal records. It is important to note that among these multiple dimensions we can recognize groups that are highly related among themselves, but remain quite different from other groups. For example, there exists a high conceptual affinity among the three spatial dimensions $(x, y, z)$ as they are represented by similar structures and often have comparable values, while there is an obvious lack of such affinity among them and an alphanumeric ownership record. Accordingly, dimensions tend to be grouped together in conceptual features (e.g. spatial information, thematic attributes).

However, regardless of conceptual affinity, heterogeneous features may display high dependency among them (e.g. space and time). This dependency needs to be exploited when querying a database in order to recognize for example complex spatiotemporal events and patterns. Querying space and time separately would fail to adequately address this inherent spatiotemporal complexity. Similarly, the radiometric content of satellite imagery may be highly correlated to sensor information. This grouping of dimensions and the need to exploit cross-grouping dependencies is another issue that differentiates geospatial databases from other high-dimensional ones.

3) Data volume. Like many disciplines where learning algorithms are applied, GIS is rich in data. In addition to traditionally considered geospatial databases (e.g. maps, photographs), numerous other databases (e.g. consumer, medical, and financial records) are now incorporating spatial and temporal attributes and hence offer the possibility of discovering or confirming geographical knowledge [15]. As mentioned above, geospatial dataset collections are now terabyte-sized, and traditional retrieval methods have a hard time to keeping up. Furthermore, maintaining and evaluating these large amounts of information is a major challenge, leading to frequent occurrence of incomplete or missing data.

4) Complexities due to local variation. Earth systems are so intrinsically interconnected that it is difficult to isolate an analysis conducted on some part of a system from the affects of other unmodeled aspects [8]. This translates into potential generalization problems of similarity algorithms. Measured geographic attributes often exhibit the seemingly contradictory properties of spatial correlation and spatial heterogeneity. The former (correlation) refers to the tendency of attributes at some locations in space to be related, also known as Tobler's first law of geography [16]: "Everything is related to everything else but nearby things are more related than distant things”. However, and despite the effect of spatial corre- 
lation on the major trends of spatial information, geographic phenomena are often highly localized. Spatial heterogeneity describes this non-stationarity of most geographic processes, and expresses the fact that global parameters do not necessarily describe well the localized nature of some geographical phenomena.

5) Granularity. In most non-geographic domains, data objects are meaningfully represented discretely within the information space without losing important properties [17]. But this does not seem to extend to geographic objects [18]: size, shape and boundaries can affect geographic processes, therefore generalization cannot be achieved without information loss in both raster and vector representations. Scales and granularities for measuring time are also complex, preventing a simple “dimensioning up" of space to include time. Moreover micro data, observations on individual observational units, might not always be accessible, e.g. due to dissemination, confidentiality or cost constraints. Macro data (aggregates of micro data) are used instead. Examples of macro data include counts, frequencies, sums, averages and other statistics characterizing micro data.

\section{Geospatial User Profiles}

\subsection{Motivation}

Until now we defined desired characteristics for a similarity learning algorithm. Similarity is typically calculated by comparing a stored set of values to the ones the users query for. First each query value (attribute) is compared to the corresponding stored one, for example the time of a stored aerial photograph to the corresponding query value for time, the scale of the stored aerial photograph to the query and so on for every requested attribute. Then results from this comparison expressing similarity within every attribute (similarity in time, scale, etc) are aggregated to provide an overall similarity metric, a metric showing the overall similarity between the query and the stored aerial photograph based on these individual metrics from every attribute.

Existing methodologies concentrate on multi-attribute (i.e. multi-dimensional) similarity aggregation to provide an overall similarity metric. In some cases though problem complexity relies on the similarity calculation within each dimension separately rather than on their combined aggregation. This is frequently the case when querying for GIS datasets. The information retrieval process might fail because the individual similarity metrics in every dimension may not be able to capture user similarity preferences.

A common example of such similarity preference in GIS is when asymmetric, non-linear user behavior is exhibited during the direct comparison of attributes. For example, let us consider a geospatial database and a user request for an aerial image of specific ground pixel size for building extraction. User interest decreases gradually (but not necessarily linearly) as pixel size increases to the degree that buildings would not be identifiable. Furthermore, the user may have cost considerations (e.g. cost, storage and processing time) associated with a higher resolution acquisition. This translates to a similarity relation that can also be non-linear as resolution improves. So it is easily understood that we need asymmetrical, non-linear relations to model user preference within each attribute comparison. Thus, in geospatial queries user preferences may be significantly more complex than general queries (e.g. text queries), while the diversity of users and applications is further emphasizing the need for efficient modeling. Therefore, modeling user similarity preference within each attribute can substantially help geospatial queries. Motivated by these observations, the focus of our work is to investigate the application of complex functions for user preference within each attribute. The integration of similarity results from multiple attributes is part of our future work.

\subsection{A User Preference-Based Approach}

In order to adapt similarity models to user preferences we developed a relevance feedback algorithm. Users are presented with a variety of pairs of requested and returned values and are asked to provide a preference metric for each pair. The corresponding training dataset is created and used as input for our preference learning method. Figure 2 shows a typical training session, where the user is given the Query ( $X$ axis), and Database value ( $Y$ axis) and is requested to provide a similarity assessment of these two.

The result corresponds to the Similarity value ( $Z$ axis). The problem can easily be seen as a surface-fitting one,

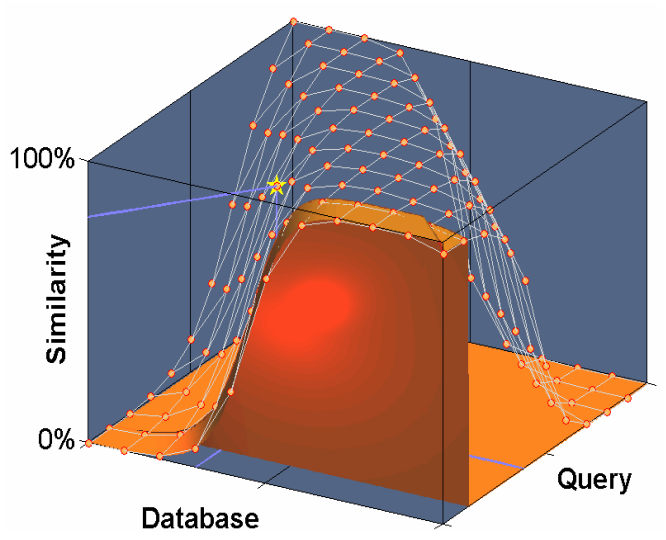

Figure 2. Training example. 
where it is attempted to substitute the provided threedimensional points with a surface (function). For training several preference models are used of as expressed through a variety of fuzzy membership functions (FMFs). The approach is simple yet effective: gradually increase the complexity of the underlying FMF until an acceptable solution is reached. The process begins by interpolating a set of planes to the training dataset [19]. We examine the resulting accuracy and if it is within the predefined specifications we end the process. These predefined specifications are in essence thresholds describing the maximum acceptable error between the interpolated functions and the training points. They can be preset by the database designer or adjusted in real-time by the user. If the results are not within these thresholds, we examine the obtained plane parameters. This analysis leads to a decision whether similarity is dependent on the query value, their difference metric or the actual database and query values. We continue by interpolating two sigmoidal functions whose initial approximations are calculated from the plane properties. If required accuracy is not achieved, we provide further modeling capabilities by parameterizing further the FMFs parameters. At the last stage we obtain the best possible set of FMFs that express user preference as presented through the training set. If accuracy is not yet achieved, we trigger a neural network process to correct local errors. More information on the training mechanism and the corresponding modeling capabilities can be found in [19].

After the best possible set of functions is identified, the mathematical properties of the model are stored in the form of a profile. This profile can also contain a User
ID, and potentially comments/keywords that will allow usability of the same profile from other users to avoid retraining the system. For example, such keywords might be general such as "Photogrammetrist" or "Biologist", or more task-specific such as "Airplane feature extraction", "Wetland evaluation".

To further demonstrate the applicability of the method a representative example is presented below for a cadastre/real estate application. More specifically, this scenario investigates user preference of a geospatial attribute expressing parcel value per square meter. The function is composed of two sub-functions, each one applicable in half of the input space (e.g. $X_{q}>X_{d b}$ ) to compensate for asymmetrical cases. A result of this trained function can be seen in Figure 3.

Figure 4 shows similarity isolines ( $0 \%$ to $100 \%$ at the graph floor) of the surface from Figure 3, in essence combinations of query and database values that would result in the same similarity value. In addition, two specific user queries are examined through the two slices, for parcel value per square meter (PVSM) of $\$ 500 / \mathrm{m}^{2}$ (in orange) and $\$ 3000 / \mathrm{m}^{2}$ (in green). Examination of these two sections leads to two remarks:

1) The left side of each of the two sections examines the case where the returned PVSM value $\left(X_{d b}\right)$ is smaller than the query PVSM value $\left(X_{q}\right)$. Here the method is able to express the gradual decrease of user's interest. Note in Figure 4 how user flexibility increases as the PVSM query value becomes larger.

2) The right side of each of the two sections examines the case where the returned PVSM value $\left(X_{d b}\right)$ is larger than the query PVSM value $\left(X_{q}\right)$. From the two sections

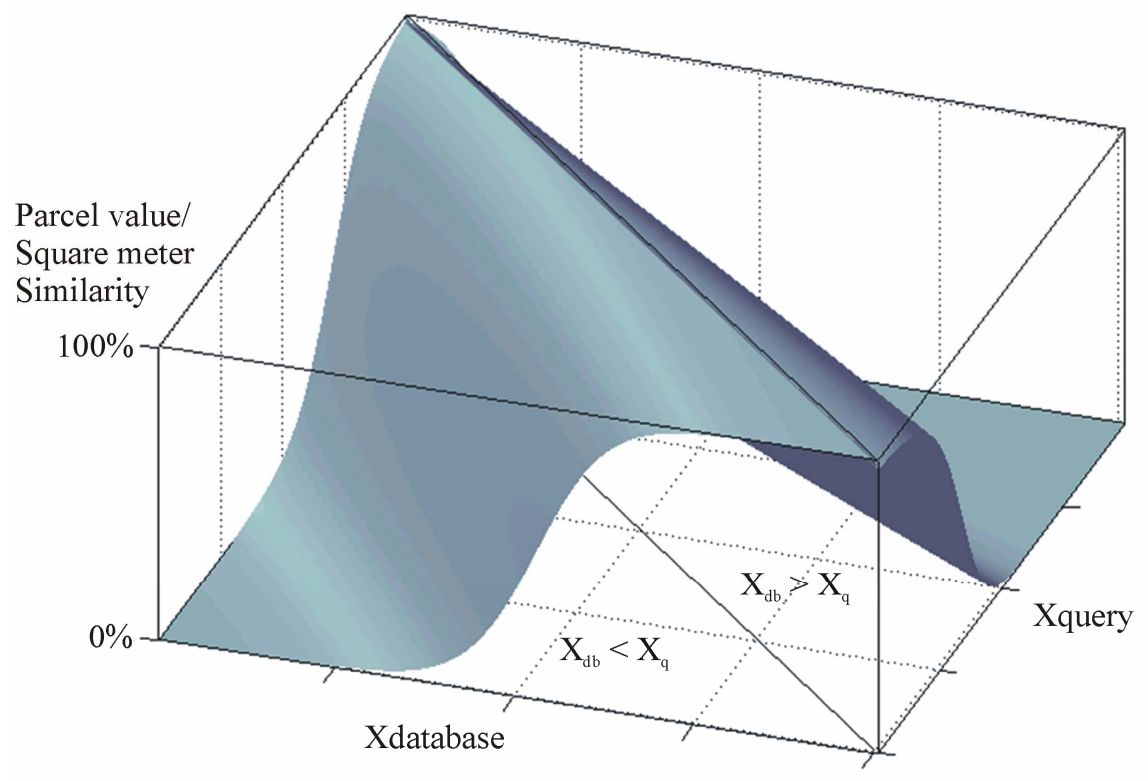

Figure 3. Example of a user preference function. 


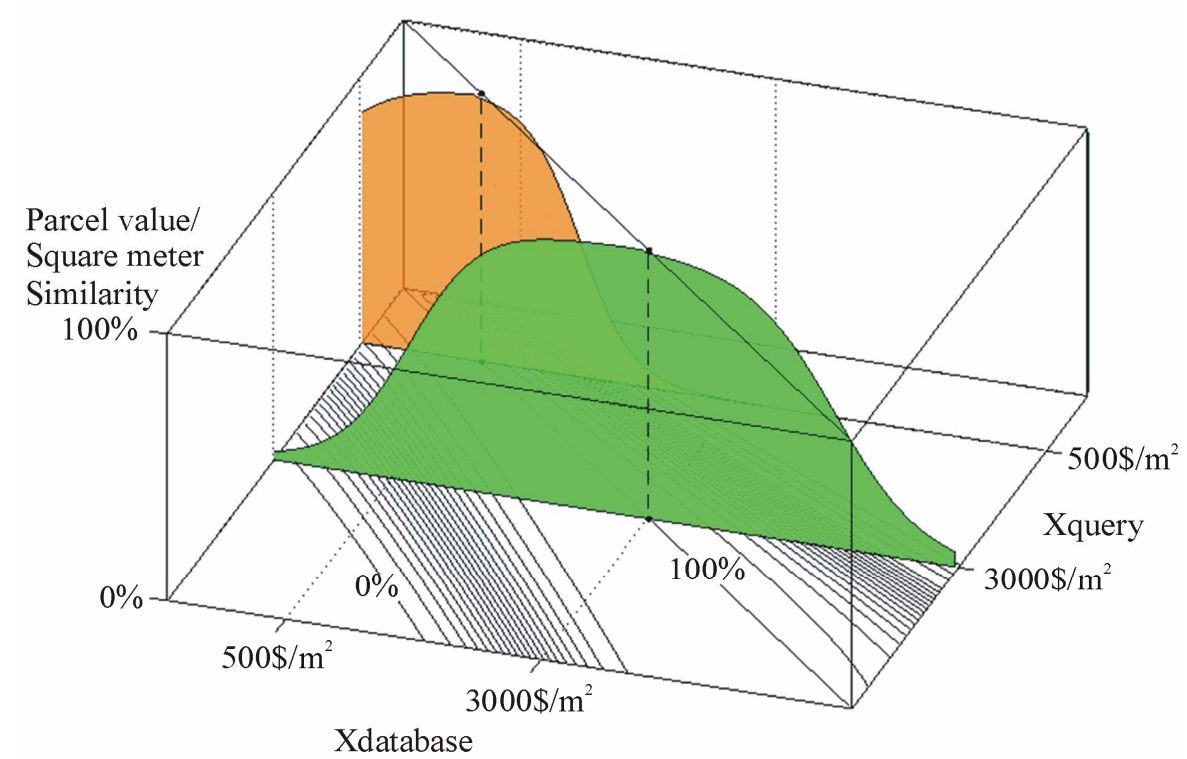

Figure 4. Contour plot and query examples of this preference function.

it is evident that as the query PVSM value $\left(X_{q}\right)$ increases so does the user flexibility on the obtained results. More specifically, when users request the retrieval of database objects with $\$ 500 / \mathrm{m}^{2}$ PVSM they are less flexible in accepting larger values than when querying for a $\$ 3000 / \mathrm{m}^{2}$ one.

\section{Using Profiles in Queries}

In order to demonstrate the applicability of our method, let us consider the following scenario. The City of Tempe had cameras installed to monitor its downtown area. Numerous city agencies use this information for their various needs. For example, let's consider that imagery from these cameras is accessed by both the Police and Transportation Departments. Let's also assume that they perform similar queries, using last year's New Year's Eve imagery database to train personnel in anticipation of this year's celebrations. They are interested in recovering an image of the downtown area at 12 midnight, to get a snapshot of the situation, so they form a query to express this request. Even though they form the same query, the execution of this query proceeds differently for these two agencies, making use of their preferences as they are expressed through corresponding profiles. Algorithm training is performed based on established similarity preferences, and the corresponding similarity profiles are shown in Figures $\mathbf{6}$ and $\mathbf{7}$ for the Police and Transportation Departments, respectively. For comparison we also present a generic profile in Figure 5.

By using these different profiles in the query processing it is feasible to rank available imagery differently, taking into account their different needs. For example, the Police profile has the following main characteristics:

- The time interval $11 \mathrm{pm}-12$ am is of prime importance, as this is the instance with the highest crowd concentration and overall activity.

- After 12 midnight interest begins dropping, as people start leaving, but remains high until $3 \mathrm{am}$.

On the other hand, the Transportation profile has some other characteristics:

- Its peak is around $12 \mathrm{am}$, when people (potentially intoxicated) start leaving the area, posing a higher risk of accidents.

- Early on, interest is increasing as we move from the standard traffic patterns of $9 \mathrm{pm}$ to higher traffic loads by $10: 45 \mathrm{pm}$.

- Interest drops between 10:45 pm and 11:15 pm, as by that time people have already arrived, and thus vehicle traffic is limited. It starts picking up again after 11:30 pm as few people may be leaving earlier.

A sample of 5 images has been ranked, to demonstrate the effects of user preferences. This is shown in Figure 8. For example, that imagery from 11:20 pm is ranked first for the police department, even though it deviates from the query request (midnight) by 40 minutes, when there is an image with only 15 minutes away from the query time (12:15 am). However, for the above mentioned reasons the 11:20 pm is more suitable for this department's needs than the 12:15 am snapshot. Other rankings have similar explanations based on the above mentioned special preference characteristics as expressed through the corresponding profile. It is obvious that generic profiles could not express such diverse similarity preference pat 


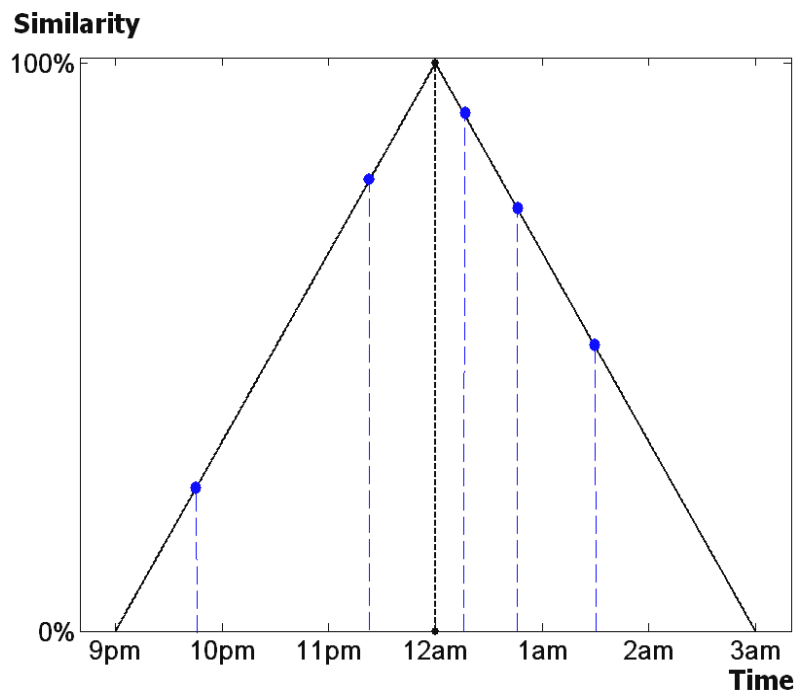

Figure 5. Generic similarity profile.

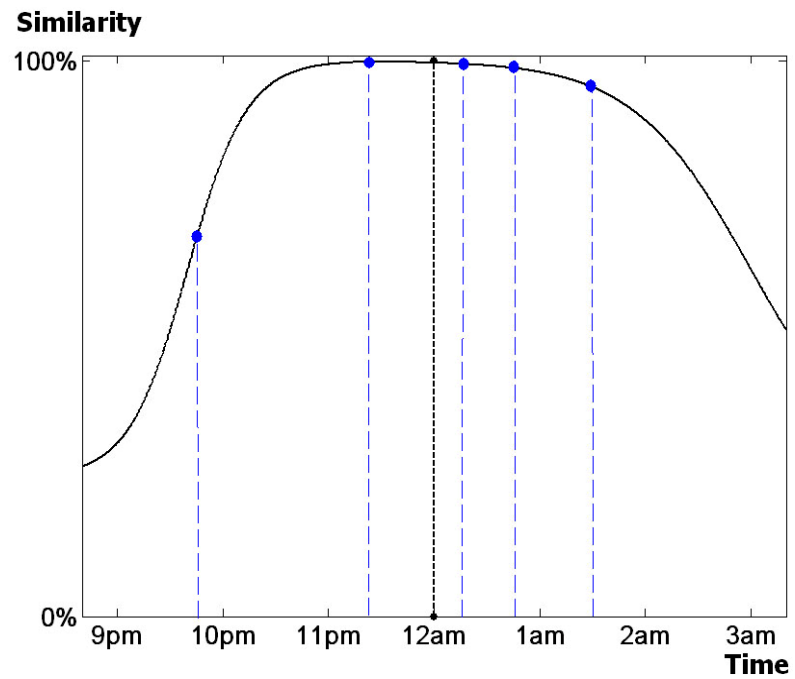

Figure 6. Police surveillance similarity profile.

terns, limiting the effectiveness of query-based information retrieval.

\section{Conclusions}

Geospatial datasets are becoming increasingly multifunctional, as different users may be using the same dataset for different applications. Accordingly, the successful functional integration such datasets in federated geospatial databases depends upon the ability to meet the needs of expanding and diverse user communities. Therefore, the development of efficient information retrieval methods to support the diverse and complicated preference patterns of different users and/or applications is a crucial task for the geoinformatics community.

In this paper we presented an approach to meet this

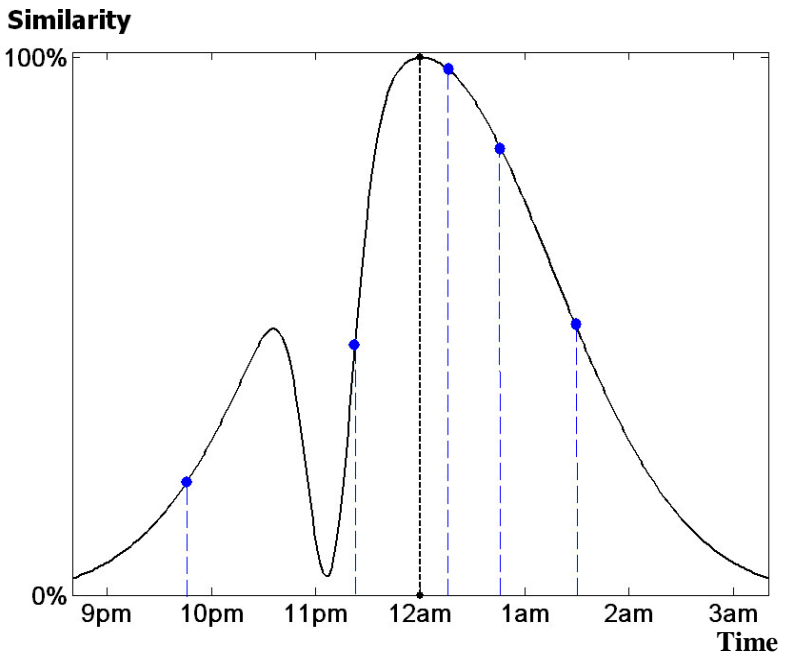

Figure 7. Traffic monitoring similarity profile.

need through the introduction of user profiles of varying complexity to model the requirements of different classes of users when attempting to recover specific geoinformation. Intelligent systems can assist geospatial queries to improve retrieval accuracy by customizing results based on preference patterns. The profiles may vary in their complexity, thus capturing the underlying preference intricacies that differentiate user groups (e.g. the needs of a transportation expert versus the ones of a police authority).

As presented in this paper, our method emphasizes preference modeling within specific attributes (e.g. preferences in time, scale, resolution). Our future plans include the extension of this work to aggregate these individual components into composite multidimensional user profiles. Depending on the application range of a specific government agency, these composite profiles may reflect preferences of a single analyst or of a broader unit with a specific mission and modus operandi.

While user preference profiles were introduced in this paper as a tool to support information retrieval tasks, they also encapsulate operational knowledge: they are expressions of a user's typical tasks and processes. Accordingly, we can recognize a very intriguing indirect benefit of our approach, namely the ability to identify similarities in user communities that may be operationally different. For example, by comparing user profiles between groups of analysts from an environmental and an emergency response agency we may reach the conclusion that they have comparable preferences and tend to perform similar tasks. This information can be used for operational alignments across different units/agencies. Furthermore, preference profiles may be used to prioritize data collection and information acquisition needs. Types of datasets that exhibit high similarity preference 


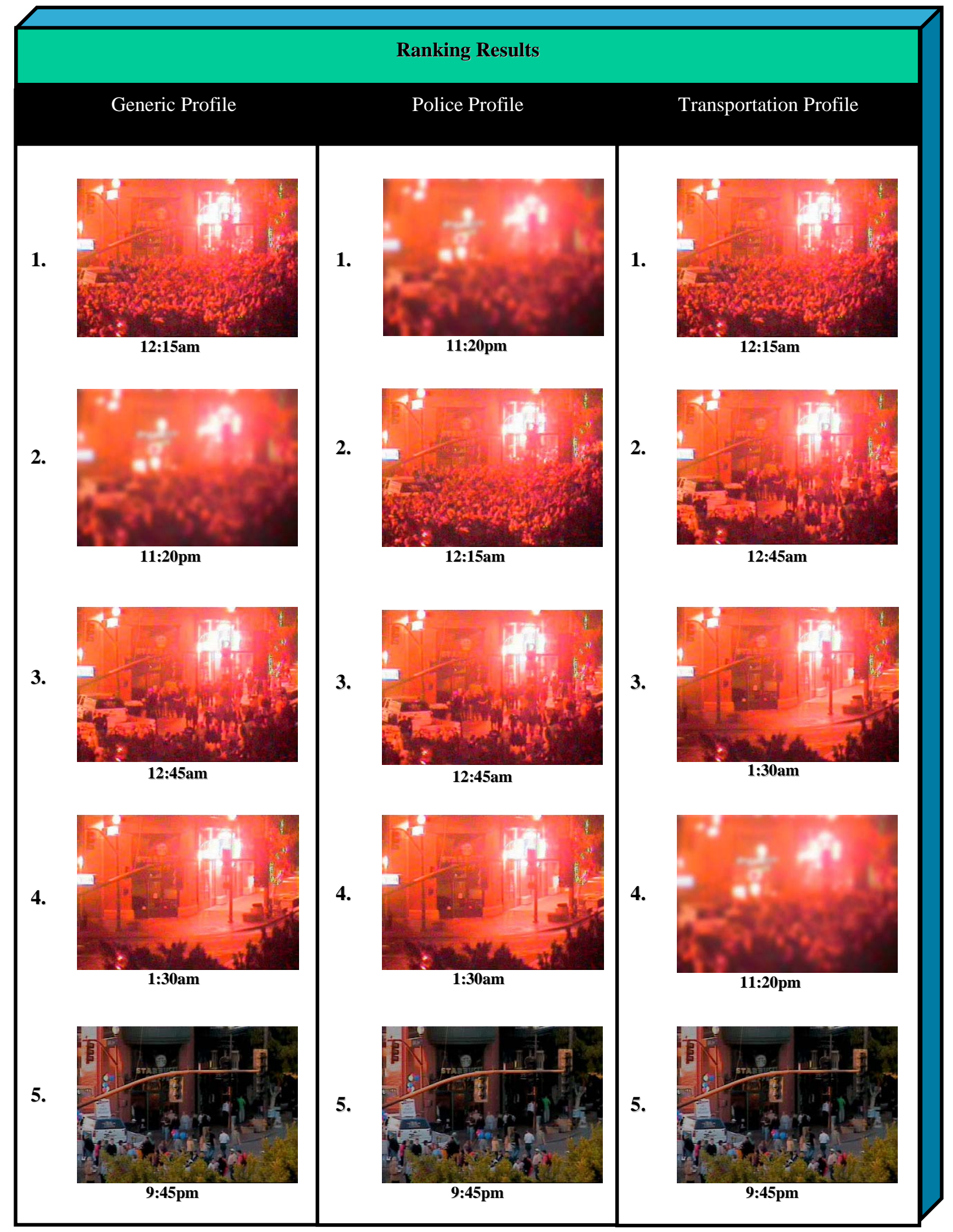

Figure 8. Effects of profiles on geospatial query results (images from www.tempe.gov). 
across numerous profiles should be updated more frequently than others with lower priority. Combined with the above mentioned capability to identify across agencies clusters of users with similar needs and preferences, this would provide crucial support for the reconfiguration of government resources to best address evolving needs and emerging challenges.

\section{References}

[1] M. F. Goodchild, "Citizens as Sensors: The World of Volunteered Geography,” GeoJournal, Vol. 69, No. 4, 2007, pp. 211-221. doi:10.1007/s10708-007-9111-y

[2] V. M. Megler and D. Maier, "Finding Haystacks with Needles: Ranked Search for Data Using Geospatial and Temporal Characteristics. Scientific and Statistical Database Management," Scientific and Statistical Database Management, Vol. 6809, 2011, pp. 55-72. doi:10.1007/978-3-642-22351-8_4

[3] D. Sui, "The Wikification of GIS and Its Consequences: Or Angelina Jolie's New Tattoo and the Future of GIS," Computers, Environment, and Urban Systems, Vol. 32, No. 1, 2008, pp. 1-5. doi:10.1016/j.compenvurbsys.2007.12.001

[4] S. Liu and A. Iacucci, "Crisis Map Mashups in a Participatory Age," American Congress on Surveying and Mapping Bulletin, 2010, pp. 10-14.

[5] D. W. Aha, D. F. Kibler and M. K. Albert, "InstanceBased Learning Algorithms,” Machine Learning, Vol. 6, No. 1, 1991, pp. 37-66. doi:10.1007/BF00153759

[6] W. Cheng and E. Huellermeller, "Combining InstanceBased Learning and Logistic Regression for Multilable Classification,” Machine Learning, Vol. 76, No. 2-3, 2009, pp. 211-225. doi:10.1007/s10994-009-5127-5

[7] P. Cunningham, "A Taxonomy of Similarity Mechanisms for Case-Based Reasoning," IEEE Transactions on Knowledge and Data Engineering, Vol. 21, No. 11, 2009, pp. 1532-1543. doi:10.1109/TKDE.2008.227

[8] B. Batchelor, "Pattern Recognition: Ideas in Practice," New York Plenum Press, New York, 1978, pp. 71-72.

[9] D. R. Wilson and T. R. Martinez, “An Integrated Instance-Based Learning Algorithm,” Computational Intel- ligence, Vol. 16, No. 1, 2000, pp. 1-28. doi:10.1111/0824-7935.00103

[10] G. Mountrakis, P. Agouris and A. Stefanidis, "Similarity Learning in GIS: An Overview of Definitions, Prerequisites and Challenges,” In: M. Vassilakopoulos, A. Papadopoulos and Y. Manolopoulos, Eds., Spatial Databases: Technologies, Techniques and Trends, Idea Group Inc., Calgary, 2004, pp. 294-321. doi:10.4018/978-1-59140-387-6.ch013

[11] D. Gunopulos, “Data Mining Techniques for Geospatial Applications," National Academies White Paper, 2001.

[12] M. Gahegan, "Intersection of Geospatial Information and Information Technology," National Academies White Paper, 2001.

[13] National Research Council, "Distributed Geolibraries: Spatial Information Resources,” National Academy Press. Washington, DC, 1999.

[14] A. S. Camara and J. Raper, "Spatial Multimedia and Virtual Reality,” Taylor \& Francis, London, 1999.

[15] H. J. Miller and J. Han, "Geographic Data Mining and Knowledge Discovery: An Overview,” In: H. J. Miller and J. Han, Eds., Geographic Data Mining and Knowledge Discovery, Taylor and Francis, London, 2001. doi:10.4324/9780203468029_chapter_1

[16] W. Tobler, “Cellular Geography,” In: S. Gale and G. Olsson, Eds., Philosophy in Geography, Reidel, Dortrecht, 1979, pp. 379-386.

[17] M. Yuan, B. Buttenfield, M. Gahegan and H. Miller, "Geospatial Data Mining and Knowledge Discovery," A UCGIS White Paper on Emergent Research Themes, 2001 http://www.ucgis.org/emerging/

[18] J. Lin, Y. Fang, W. Zhang and Z. Huang, "Fundamental Aspects of Access Control for Geospatial Data," International Journal of Digital Earth, Vol. 2, No. 3, 2009, pp. 275-289. doi:10.1080/17538940902818329

[19] G. Mountrakis and P. Agouris, "Learning Similarity with Fuzzy Functions of Adaptable Complexity. 8th International Symposium on Spatial and Temporal Databases," Lecture Notes in Computer Science, Vol. 2750, 2003, pp. 412-429. doi:10.1007/978-3-540-45072-6_24 